\title{
Reflexiones en torno a la "prostitución" como trabajo sexual y su relación con el delito de trata de personas con fines de explotación sexual y la violencia de género
}

Reflections on "prostitution" as sex work and its relation to the crime of trafficking in persons for the purpose of sexual exploitation and gender-based violence

Reflexões sobre "prostituição" como trabalho sexual e sua relação com o crime de tráfico de pessoas para fins de exploração sexual e violência de gênero Réflexions sur la "prostitution" en tant que travail du sexe et ses relations avec le crime de traite des personnes à des fins d'exploitation sexuelle et de violence de genre

Lorena Ríos $^{1}$ | Universidad Nacional de La Plata

Revista Derechos en Acción / ISSN 2525-1678 / e-ISSN 2525-1686 Año 4/NNo 10 Verano 2018/2019 (21 diciembre a 21 marzo), 234-248 DOI: https://doi.org/10.24215/25251678e252

ORCID: https://orcid.org/0000 000172618545

Recibido: 17/02/2019

Aprobado: 01/03/2019

Resumen: El delito de trata de personas con fines de explotación sexual, la "prostitución" y la violencia de género son conceptos íntimamente relacionados, que tienen su origen en la desigualdad de género, pero que también tienen existencia individual, es decir, pueden presentarse sin estar relacionados entre ellos, con lo cual constituyen situaciones

1 Abogada egresada de la Facultad de Ciencias Jurídicas y Sociales de la UNLP, integrante del Ministerio Público de la Nación. Magister en Derechos Humanos, Facultad de Ciencias Jurídicas y Sociales de la UNLP. 
jurídicas que merecen un análisis completo acorde a sus particularidades, para evitar confusiones y evitar la aplicación automática de razonamientos equivocados. Por ello en el presente trabajo brindaré algunas definiciones a dichos conceptos para luego poder fundamentar dicha afirmación.

Previo al desarrollo de mi argumentación, cabe aclarar que un análisis completo de la problemática planteada debería incluir además de aspectos jurídicos, aspectos psicológicos, sociológicos, culturales y económicos entre otros. Sin embargo, teniendo en cuenta el alcance del presente trabajo, sólo se desarrollará desde una perspectiva jurídica, señalando que resulta válido el aporte aunque sea parcial ya que podrá sumarse a posteriores análisis interdisciplinarios que confluyan a confirmar las conclusiones que se obtendrán.

Palabras claves: trata de personas; prostitución; violencia de género.

Abstract: The crime of trafficking in persons for the purpose of sexual exploitation, "prostitution" and gender-based violence are intimately related concepts, which have their origin in gender inequality, but which also have an individual existence, that is, they can be presented without being related to each other, and therefore constitute legal situations that deserve a complete analysis according to their particularities, in order to avoid confusion and the automatic application of mistaken reasoning. For this reason, in this paper I will provide some definitions of these concepts in order to be able to support this affirmation.

Prior to the development of my arguments, it should be stated that a complete analysis of the proposed problem should include, in addition to legal aspects, psychological, sociological, cultural and economic aspects, among others. However, taking into account the scope of the present article, it will only be developed from a legal perspective, pointing out that the contribution is valid even if it is partial since it can be added to subsequent interdisciplinary analyses that converge to confirm the conclusions that will be obtained.

Keywords: trafficking in persons; prostitution; gender-based violence.

Resumo: 0 crime de tráfico de pessoas com fins de exploração sexual, a "prostituição" e a violência de gênero são conceitos intimamente relacionados, que têm sua origem na desigualdade de gênero, mas também têm 
existência individual, é dizer, podem apresentar-se sem estarem relacionados entre si, com o qual constituem situações jurídicas que merecem uma análise completa de acordo com suas particularidades, para evitar confusões e evitar a aplicação automática de raciocínio errado. Por isso, no presente trabalho vou dar algumas definições a esses conceitos para logo poder basear essa afirmação.

Antes do desenvolvimento da minha argumentação, deve-se esclarecer que uma análise completa da problemática tratada deveria incluir além de aspectos jurídicos, aspectos psicológicos, sociológicos, culturais e econômicos, entre outros. No entanto, levando em conta o escopo deste trabalho, só se desenvolverá desde uma perspectiva legal, apontando que é válida a contribuição embora seja parcial já que podará ser adicionada às análises interdisciplinares subsequentes que convirjam a confirmar as conclusões que se obtenerão.

Palavras-chave: tráfico de pessoas; prostituição; violência de gênero.

Résumé: Le crime de traite des personnes à des fins d'exploitation sexuelle, la "prostitution" et la violence de genre ce sont des concepts interdépendants, qui ont leur origine dans l'inégalité de genre mais ils ont aussi une existence individuelle, c'est-à-dire qu'ils peuvent être présentés sans être liés les uns aux autres. Ces concepts constituent des situations juridiques qui méritent une analyse complète en fonction de ses particularités, afin d'éviter toute confusion et l'application automatique d'un mauvais raisonnement. Par conséquent, dans le présent travail, je fournirai quelques définitions de ces concepts pour pouvoir fonder cette affirmation. Avant de développer mon argumentation, il convient de préciser qu'une analyse complète des questions soulevées devrait également inclure les aspects juridiques, ainsi que les aspects psychologiques, sociologiques, culturels et économiques. Cependant, compte tenu de la portée de ce travail, il ne sera développé que d'un point de vue juridique, notant que la contribution est valable même si elle est partielle, dans la mesure où il pourrait être ajouté aux analyses interdisciplinaires ultérieures qui convergent pour confirmer les conclusions qui seront obtenues.

Mot-clés: traite des personnes- prostitution- la violence de genre 


\section{Introducción}

El delito de trata de personas con fines de explotación sexual, la "prostitución" y la violencia de género son conceptos íntimamente relacionados, que tienen su origen en la desigualdad de género, pero que también tienen existencia individual es decir, pueden presentarse sin estar relacionados entre ellos, con lo cual constituyen situaciones jurídicas que merecen un análisis completo acorde a sus particularidades, para evitar confusiones y evitar la aplicación automática de razonamientos equivocados. Por ello en el presente trabajo brindaré algunas definiciones a dichos conceptos para luego poder fundamentar dicha afirmación.

Previo al desarrollo de mi argumentación, cabe aclarar que un análisis completo de la problemática planteada debería incluir además de aspectos jurídicos, aspectos psicológicos, sociológicos, culturales y económicos entre otros. Sin embargo, teniendo en cuenta el alcance del presente trabajo, sólo se desarrollará desde una perspectiva jurídica, señalando que resulta válido el aporte aunque sea parcial ya que podrá sumarse a posteriores análisis interdisciplinarios que confluyan a confirmar las conclusiones que se obtendrán.

\section{Patriarcado y violencia de género}

La violencia de género fue definida por la Declaración sobre la eliminación de violencia contra la mujer proclamada por la Asamblea General de las Naciones Unidas en el año 1993 en su art. $1^{\circ}$ como:

"todo acto de violencia basado en la pertenencia al sexo femenino que tenga o pueda tener como resultado un daño o sufrimiento físico, sexual o sicológico para la mujer, así como las amenazas de tales actos, la coacción o la privación arbitraria de la libertad, tanto si se producen en la vida pública como en la vida privada."

2 Entrecomillo el término por considerarlo que tiene una connotación negativa para quienes ofrecen trabajos sexuales. 
Luego, en el art. 2, se enumeran una serie de actos de violencia no taxativos:

“... a) La violencia física, sexual y sicológica que se produzca en la familia, incluidos los malos tratos, el abuso sexual de las niñas en el hogar, la violencia relacionada con la dote, la violación por el marido, la mutilación genital femenina y otras prácticas tradicionales nocivas para la mujer, los actos de violencia perpetrados por otros miembros de la familia y la violencia relacionada con la explotación; b) La violencia física, sexual y sicológica perpetrada dentro de la comunidad en general, inclusive la violación, el abuso sexual, el acoso y la intimidación sexuales en el trabajo, en instituciones educacionales y en otros lugares, la trata de mujeres y la prostitución forzada; c) La violencia física, sexual y sicológica perpetrada o tolerada por el Estado, dondequiera que ocurra."

La desigualdad de género, que conlleva a la violencia ejercida sobre las mujeres, en sus distintas formas y dimensiones, no es algo novedoso. Es bueno recordar que las mujeres históricamente han tenido que luchar para poder ser titulares de derechos y luego han tenido que seguir luchando para poder ejercer dichos derechos reconocidos normativamente. Ejemplos hay muchos, pero sólo para nombrar uno emblemático recordemos a Olympe de Gouges, francesa feminista escritora y abolicionista, quien en el año 1791 redactó la Declaración de Derechos de la Mujer y de la Ciudadana parafraseando la Declaración de los Derechos del Hombre y del Ciudadano y dos años después, en 1793, fue guillotinada.

A nivel internacional y regional no fueron suficientes los instrumentos generales que suponían la inclusión de todas las personas $^{3}$; sino que las mujeres debieron luchar por instrumen-

\footnotetext{
3 Declaración Universal de Derechos Humanos; Pacto Internacional de Derechos Civiles y Políticos; Pacto Internacional de Derechos Económicos, Sociales y Culturales; Declaración Americana de los Derechos y Deberes del Hombre; Convención Americana sobre Derechos Humanos y el Protocolo a la Convención Americana, entre otros.
} 
tos específicos para dejar claro que también eran sujetas de derechos, dictándose entre otros instrumentos la Convención sobre la eliminación de todas las formas de discriminación contra la mujer y su protocolo facultativo; la Declaración sobre la eliminación de la violencia contra la mujer y la Convención Interamericana para Prevenir, Sancionar y Erradicar la Violencia Contra la Mujer.

La violencia de género es un flagelo mundial que lleva años de lucha intentando ser combatida. Se ha intentado dar un marco jurídico para solucionar dicha problemática, pero siendo la misma un tema que excede lo jurídico y encontrándose implicadas cuestiones culturales y sociales, entre otras, hacen falta más que respuestas jurídicas para solucionar de manera integral la temática. Ello porque, siguiendo a Fraser (1997), las injusticias que reflejan tanto las luchas por la redistribución como las luchas por el reconocimiento encuentran su origen en la estructura industrial del capitalismo basada en un orden de género, en el que el hombre era el único proveedor y la mujer la única cuidadora. En ese sentido Fraser sostiene que:

"Se suponía que las personas estaban organizadas en familias nucleares beterosexuales encabezadas por un hombre, cuya principal fuente de ingreso era el salario del hombre en el mercado de trabajo. El hombre cabeza de familia recibía un salario familiar, esto es, un salario suficiente para mantener a los bijos y a una esposa-y-madre de tiempo completo, quien se dedicaba al trabajo doméstico de tiempo completo, sin recibir ninguna remuneración. Desde luego, las vidas de muchas personas nunca se ajustaron a este patrón; aun así, ésta era la representación normativa de lo que debía ser la familia correcta." (Fraser, 1997:56).

En el caso en que las mujeres no se dedicaran a tiempo completo al ámbito doméstico, los trabajos que les fueron asignados culturalmente fueron y son aún hoy, remunerados con salarios más bajos que los asignados culturalmente a los hombres. 
"¿Por qué las mujeres tenían el mandato "natural" de quedarse en el hogar? Porque la posibilidad de reproducción biológica de las mujeres (concepción, embarazo, parto y lactancia) se relacionó como algo inescindible con las tareas de cuidado y crianza, naturalizándose de ese modo dichas tareas." (Ríos, 2017:75)

De este modo las mujeres además de luchar por el reconocimiento de una identidad cultural deben luchar por la igualdad social. Estas luchas, que posicionan a la mujer de un modo diferente en el espacio público y político, han despertado la molestia, decodificada en violencias de todo tipo ${ }^{4}$, de muchos que no quieren ser desplazados de dichos espacios y que no quieren que la mujer ocupe otro lugar que el rol que se le ha asignado históricamente, ya que esto para el sistema capitalista y patriarcal, sería una catástrofe.

\section{La trata de personas con fines de explotación sexual}

La trata de personas, especialmente de mujeres y niñas, es uno de los delitos actuales más atroces a los que se debe enfrentar la comunidad internacional. En ese sentido tal como destaca Kofi A. Annan en el prefacio de la Convención de las Naciones Unidas contra la delincuencia organizada transnacional estamos ante un fenómeno que cada vez se agrava más, basado en prácticas discriminatorias de género y aprovechándose de las condiciones sociales y económicas que atraviesan los países de origen de las víctimas que sufren este flagelo.

\footnotetext{
4 Como sostiene Segato "se pasa por alto que todas esas violencias a "minorías" no son otra cosa que el disciplinamiento que las fuerzas patriarcales nos imponen a todos los que habitamos ese margen de la política. Se trata de crímenes del patriarcado colonial moderno de alta intensidad, contra todo lo que lo desestabiliza, contra todo lo que parece conspirar y desafiar su control, contra todo lo que se desliza hacia fuera de su égida, con las varias estrategias y tácticas diarias con las que muchos de nosotros, a propósito o inadvertidamente, nos deslizamos y escabullimos de la vigilancia patriarcal y la desobedecemos. Expurga de ese modo todo lo que no le concede el reconocimiento debido a su forma de estructurar y disciplinar la vida, a su forma de habilitar y naturalizar un camino de asimetrías y dominaciones progresivas." (Segato, 2016:96)
} 
La trata de personas es una violación grave a los derechos humanos que afecta a millones de personas, en especial mujeres, niños y niñas.

Este crimen ha generado y sigue generando preocupación a nivel mundial razón por la cual se han tomado una serie de medidas al respecto en el marco de la Organización de Naciones Unidas.

Durante la Conferencia Mundial de Derechos Humanos realizada en Viena en el año 1993 se determinó que "la violencia y todas las formas de acoso y explotación sexuales, en particular las derivadas de prejuicios culturales y de la trata internacional de personas son incompatibles con la dignidad y la valía de la persona humana y deben ser eliminadas."

El delito de trata de personas ${ }^{5}$, en tanto presupone la finalidad de explotación, no es nuevo sino que es el resurgir de antiguas prácticas. La esclavitud era el supuesto de cosificación más extremo que la humanidad haya tenido conocimiento. Esta aberración, en la antigüedad tuvo una aceptación prácticamente total, nótese que hasta los estados más modernos, como Roma y Grecia y los filósofos de ideas más esclarecidas como Platón, justificaron la existencia de la esclavitud. Es decir la idea de que un ser humano podía ser objeto de comercio o intercambio y estar sometido a la absoluta voluntad de su dueño, privado de todo poder de decisión, como ha quedado demostrado, lamentablemente no es nueva.

Lo que sí es nuevo y que ha generado esta toma de conciencia de la comunidad internacional es la intensidad que ha recobrado este fenómeno en el siglo XXI, y su nueva cara o apariencia, que al no contar con el apoyo social que tuvo en la antigüedad, se realiza en forma clandestina por organizaciones

5 La figura básica del delito de trata de personas se encuentra en nuestro Código Penal en el art.145 bis el cual dispone "Será reprimido con prisión de cuatro (4) a ocho (8) años, el que ofreciere, captare, trasladare, recibiere o acogiere personas con fines de explotación, ya sea dentro del territorio nacional, como desde o hacia otros países, aunque mediare el consentimiento de la víctima." 
criminales, lo que paradójicamente aumenta sus siderales ganancias económicas.

Esta nueva apariencia de la esclavitud, consiste ya no en el uso de cadenas (si bien ha habido casos actuales), sino que se recurre a medios más sutiles, difíciles de detectar, progresivos, que resultan a la larga igual de efectivos para doblegar y hacer desaparecer la voluntad de las víctimas.

En algún momento del proceso de trata de personas la situación de violencia se presenta. Puede ocurrir al comienzo, en el momento de captación, cuando se secuestra a la víctima, o bien durante la situación denominada de ablande o al momento de llegar al lugar de destino. Sea como fuere, la violencia en el proceso de trata entra en escena en alguna oportunidad, y se perpetúa para doblegar la voluntad de la víctima, para que se someta a cumplir con lo que se le exige y también para lograr su permanencia en esa situación la mayor cantidad de tiempo posible.

Esta situación de violencia es la que genera que las personas involucradas en el delito no necesiten encerrar a las víctimas. Es decir, originan un encarcelamiento psicológico en las mismas, las cuales se encuentran en una situación de control a través de distintos métodos utilizados por los victimarios, como la servidumbre por deuda (generalmente como las víctimas carecen de recursos para el traslado al supuesto lugar de destino en donde tendrá trabajo prometido inicialmente, el costo que genera eso lo solventa inicialmente el tratante), confiscación de sus documentos, aislamiento lingüístico, de sus redes sociales y violencia física.

Ahora bien, la trata de personas, como ya se ha dicho más arriba, vulnera innumerables derechos humanos como el derecho a la integridad personal, derecho a no sufrir esclavitud o servidumbre, derecho a la libertad personal, derecho al reconocimiento de la dignidad, derecho a la salud, a estar libres de explotación, a estar libres de un trato cruel e inhumano, a estar libres de la discriminación basada en el género y a estar libres de violencias, entre otros. 
Es decir, el delito de trata de personas viola innumerables derechos humanos (vida digna, libertad, dignidad, seguridad, vida sin violencia, no discriminación, entre otros), derechos que son tal como lo sostiene Ferrajoli (1997) "indisponibles" es decir, que están sustraídos tanto a las decisiones de la política como al mercado. En virtud de su indisponibilidad activa, no son alienables por el sujeto que es su titular: no puedo vender un derecho fundamental como la libertad personal y en virtud a su indisponibilidad pasiva, no son expropiables o limitables por otros sujetos, por ello ninguna mayoría, puede privarme de la vida, de la libertad o de mis derechos de autonomía.

En virtud de ello, hay consenso internacional en combatir la trata de personas en tanto resulta ser un flagelo para la comunidad internacional.

\section{IV. ¿"Prostitutas" o mujeres trabajadoras?}

Ahora bien, no existe tal consenso con relación a la postura que se debe tomar frente al ejercicio de la "prostitución".

Existen varias definiciones de este concepto y a su vez distintos sistemas que cada país puede tener en cuenta para permitirla, tolerarla o combatirla, según decida.

Como primera aproximación al tema, resulta útil el análisis del significado del término "prostitución”, definida según la Real Academia Española, como la actividad a la que se dedica quien mantiene relaciones sexuales con otras personas, a cambio de dinero.

Siempre presupone la decisión libre y voluntaria de quien la ejerce, siendo esta una persona mayor de edad en uso pleno de sus facultades, ya que cualquier cercenamiento o limitación a la decisión del ejercicio en sí de esta actividad, nos colocaría en el campo del los delitos contra la integridad sexual, es decir no se puede practicar la "prostitución" si no es realizada libremente con el conocimiento pleno de quien realiza dicha actividad, solo así puede hablarse de que nos encontramos ante 
un acto de "prostitución". Cualquier otra práctica sexual realizada en forma gratuita o por dinero o a cambio de algo que no fuere realizada con pleno consentimiento y decidida libremente por quien la realiza, será una agresión sexual que vulnera los derechos humanos esenciales como la integridad sexual amparada en la Convención Interamericana para prevenir, Sancionar y Erradicar la Violencia contra la Mujer.

Por lo que desde un aspecto jurídico corresponde concluir que el ejercicio libre y voluntario de la prostitución, única posibilidad, desde el punto de vista conceptual, no constituye delito alguno para nuestro ordenamiento jurídico, y no debe ser utilizado como elemento valorativo para discriminar a quien realiza dicha actividad dado que debe respetarse su esfera de intimidad. ${ }^{6}$

En el orden doctrinario existen tres sistemas vinculados al ejercicio de la prostitución: Reglamentarismo, Prohibicionismo y Abolicionismo. Resumidamente, el sistema reglamentista es aquel que "tolera" y reglamenta la actividad; el prohibicionista aquel que la prohíbe y castiga y el abolicionista el que reconoce su existencia y lucha por su erradicación.

Pero tal vez desde esta sistematización, ya se arrastra la confusión de base muy común que ha quedado aclarada en el punto anterior. Estos sistemas no prestan debida atención a separar los distintos supuestos diferenciados anteriormente sino que legislan en forma general dándole a distintas situaciones el mismo tratamiento. Con lo cual se genera un reduccionismo basado en prejuicios generalizados, en los cuales se considera que el fenómeno de la prostitución es único, cuando tiene distintas manifestaciones, y contenidos como se está tratando de demostrar.

6 En definitiva, no debe confundirse el ejercicio individual de la prostitución con la explotación de la prostitución ajena y en virtud de ello, en principio, podríamos decir que no todas las mujeres que se prostituyen son explotadas por terceras personas que se quedan con un porcentaje de su actividad, Ilámeselos proxenetas o con cualquier otra denominación. La primera conducta queda dentro de la esfera de las acciones privadas de los seres humanos, en cambio la explotación de la prostitución ajena es una conducta prohibida en nuestro ordenamiento jurídico según el art. 17 de la ley 12.331, y por los arts. 125 y 126 del C.P. 
En ese sentido, actualmente los movimientos feministas han revivido una fuerte disputa que tiene sus inicios en los años '60 con la libertad sexual de las mujeres como reivindicación feminista.

A grandes rasgos, la disputa se podría resumir en dos posturas antagónicas: por un lado la postura abolicionista, en donde explotación y violencia contra las mujeres es inherente al comercio sexual y por lo tanto hay que abolir dicha práctica ya que se encuentra relacionada con la cosificación de la mujer, y la dominación del hombre sobre la misma acrecentada por un sistema capitalista cada vez mas neoliberal; y por el otro lado la postura que sostiene que la actividad del trabajo sexual tiene diferentes formas de desempeño, no necesariamente relacionadas con la dominación del hombre sobre la mujer y las prácticas de violencia sobre las mismas, por lo que el trabajo sexual debería regularse reconociéndose derechos laborales a quienes realizan dicha actividad.

Creo que esta disputa dentro de los movimientos feministas, en tanto se planteen desde posturas radicales, no va a poder zanjarse, al menos en un tiempo cercano.

Sin perjuicio de comprender la problemática que plantean ambas posturas, y sin querer dar una solución a un tema tan complejo, sino generar disparadores que puedan aportar a la discusión, creo que siempre que se han generado espacios o lugares propicios para los grandes negocios en la clandestinidad, las personas que resultan siempre perjudicadas y afectadas son las más vulnerables, en este caso, las mujeres que trabajan ofreciendo servicios sexuales.

Por otro lado, no hay que olvidar que sobre estas mujeres pesa el estigma de la doble moral. Como lo sostiene Lamas:

"La prostitución femenina produce reacciones adversas porque atenta contra el ideal cultural de castidad $y$ recato de la feminidad (Leites, 1990), y la venta de servicios sexuales ofende o irrita a muchas personas que creen que "degrada" la dignidad de la mujer. El asunto de fondo es justamente la existencia de una doble moral: 
la sexualidad de las mujeres es valorada de manera distinta de la de los hombres" (Lamas, 2016:26)

Sobre quienes pesa esta doble moral, es decir sobre las mujeres, la clandestinidad encuentra el espacio propicio para exacerbar las violencias contra ellas, ya que, en los casos en que no son víctimas de la trata de personas, se las castiga por haber osado correrse de su lugar culturalmente asignado de "mujer santa" para "elegir" ser "mujeres putas", es decir, convertirse casi en delincuentes", a la vista de la sociedad.

Entrecomillo el término "elegir" ya que tomar esa decisión en muchos casos se reduce a querer sobrevivir de algún modo ya que la otra opción que tienen muchas mujeres es quedarse en ámbitos de violencia o mucho peores, en donde no existen posibilidades laborales de ningún tipo. Digo en algunos casos porque no hay que dejar de tener en cuenta aquellas mujeres que sostienen haber elegido el trabajo sexual, y pensar de otro modo, sería desconocer su capacidad de agencia y tomar una postura paternalista sobre las mismas.

Recordemos también que los trabajos asignados culturalmente a las mujeres, en la división de roles a fines de poder llevar adelante una sociedad capitalista pensada para el hombre productor y para la mujer reproductora, fueron y son, remunerados con salarios más bajos, como ya lo señalara, que los trabajos culturalmente asignados a los hombres. ${ }^{8}$

En consecuencia, da la sensación que la preocupación por la regulación del trabajo sexual parece estar más relacionada con una cuestión de mantener el statu quo de las relaciones de

\footnotetext{
7 En ese sentido Naredo sostiene "la mujer que delinque obtiene un mayor reproche social que el hombre, pues no ha sabido comportarse conforme al rol que le viene asignado por la sociedad, por un lado sumisa, pasiva, obediente y, por otro, representante del orden familiar, mediadora de Ios conflictos ajenos, etc ... El inconsciente colectivo convierte a la mujer presa en una <antimujer>, en una madre desnaturalizada, de este modo, tiene que soportar <una doble estigmatización: primero como delincuente pero además como mujer delincuente>"(Naredo, 2012: 271).

8 Un reciente informe de la OIT (2018) da cuenta que las mujeres todavía ganan un $20 \%$ menos que los hombres.
} 
género que por la explotación en sí, en el caso en que consideráramos que la "prostitución" supone explotación.

En ese sentido me permito destacar lo sostenido por Lamas en cuento a que:

"La compra-venta de servicios sexuales está vinculada con la precariedad laboral que, más que un fenómeno transitorio, es una condición estructural del capitalismo. Por ello contrasta la preocupación escandalizada ante la "explotación sexual" de cara a la indiferencia por la explotación de las obreras, las empleadas del hogar, las campesinas, las enfermeras, las taquilleras, las meseras, las de la maquila, las barrenderas y tantas otras trabajadoras que también son explotadas." (Lamas, 2016:26,27)

$\mathrm{Y}$ aquí es donde la sociedad tiene que prestar mucha atención ante una especie de angustia moral generalizada que deriva en un pedido social de presencia estatal a través de un sistema represivo como es el sistema penal ya que en los intentos de solucionar a través del castigo penal los problemas sociales se termina criminalizando, siguiendo a Wacqwant (2004), a los que menos posibilidades de acceso a derechos tienen.

\section{Conclusiones}

Los temas analizados resultan sumamente complejos, y como lo expresé en la introducción, no se pretenden agotar los mismos, sino comenzar a aclararlos conceptualmente, para que así sirvan como punto de partida para otros análisis más profundos, alzando las voces fundamentalmente de las/os trabajadoras/es sexuales.

Quizás con la regulación del trabajo sexual como posibilidad del ejercicio libre y voluntario de la prostitución individual, se puedan dejar atrás las condiciones insalubres y la situación de vulnerabilidad de las personas que la practican, que han sido explotadas sistemáticamente en forma organizada, generando un lugar propicio en el cual se instalaron y desarrollaron las redes de tratas de personas, que aprovecharon los elementos de 
clandestinidad, corrupción, prejuicios sociales, falencias económicas y culturales, para llevar adelante su actividad.

Considerando que la igualdad es un componente fundamental de la democracia, hasta tanto no exista igualdad de oportunidades reales de género no podremos disfrutar de un estado democrático pleno.

\section{Bibliografía}

FERRAJOLI, L. (1997) Derechos y Garantías; Madrid, España. Ed. Trotta. FRASER, N. (1997). Iustitia Interrupta: Reflexiones críticas desde la posición "postsocialista", Santa Fé de Bogotá, Siglo del Hombre Editores.

LAMAS, M. (2016) Feminismo y Prostitución: la persistencia de una amarga disputa. Debate Feminista. Volumen 51, pp. 18-35. Recuperado de https://ac.els-cdn.com/S0188947816300287/1s2.0-S0188947816300287-main.pdf?_tid=54e6ed23-a892-471780eb-a0da50f6f032\&acdnat=1550780571_ab2bc9d96cab8f77a$59 \mathrm{fb} 33 \mathrm{c} 5 \mathrm{f} 654356$.

NAREDO MORELO, M. (2012). Reclusas con hijos/as en la cárcel En: $V$ LEX. En: https://app.vlex.com/\#vid/441850, pp. 263-275.

Organización de las Naciones Unidas - ONU (1993) Conferencia mundial de Derechos Humanos. Recuperado en https://www.ohchr. org/Documents/Events/OHCHR20/VDPA_booklet_Spanish.pdf

Organización Internacional del Trabajo - OIT (2018) Informe Mundial sobre Salarios 2018/2019. Recuperado de https://www.ilo.org/ global/about-the-ilo/newsroom/features/WCMS_650648/lang--es/ index.htm

RÍOS, L. (2017) Infancias como colectividades bivalentes: una mirada etnográfica sobre las representaciones sociales del "interés superior del niño/a" en contexto de encierro. Recuperado en http:// sedici.unlp.edu.ar/bitstream/handle/10915/62488/Documento_ completo__.pdf-PDFA.pdf?sequence $=1 \&$ isAllowed $=\mathrm{y}$.

SEGATO, R. (2016) La Guerra contra las mujeres. Recuperado en https://www.traficantes.net/sites/default/files/pdfs/map45_ segato_web.pdf.

WACQWANT, L. (2004). Las Cárceles de la Miseria, Buenos Aires, Manantial. 